\title{
ATRIBUTOS FÍSICO-HÍDRICOS DE UM LATOSSOLO VERMELHO EUTROFÉRRICO SOB DIFERENTES SISTEMAS DE MANEJO $\left({ }^{\mathbf{1}}\right)$
}

\author{
ORLANDO MELO DE CASTRO $\left({ }^{2}\right)$; SIDNEY ROSA VIEIRA $\left({ }^{2, *}\right)$; GLÉCIO MACHADO SIQUEIRA $\left({ }^{3}\right)$
}

\begin{abstract}
RESUMO
O objetivo deste trabalho foi avaliar o comportamento de atributos físico-hídricos de um Latossolo Vermelho eutroférrico sob diferentes sistemas de manejo. O experimento foi realizado no Centro Experimental Central do Instituto Agronômico, em Campinas, ano agrícola de 1990/1991. O solo da área é Latossolo, manejado com plantio direto (PD), preparo escarificado (PE) e preparo convencional (PC). O experimento foi realizado em parcelas de $7,5 \times 15,0 \mathrm{~m}$, com três repetições para cada sistema de manejo. Determinou-se a retenção de água, a capacidade de aeração, a capacidade de água disponível, a densidade do solo e a condutividade hidráulica do solo em função do conteúdo de água $(\theta)$, por meio do método do perfil instantâneo, avaliando-se a redistribuição da água no perfil do solo para os diferentes sistemas de manejo. Conclui-se que o preparo escarificado permitiu maior retenção de água na camada superficial, em função do aumento da porosidade; a condutividade hidráulica do solo não revelou diferenças para os três sistemas de preparo estudados na camada superficial, enquanto, em profundidade, no sistema de plantio direto ocorreram maiores valores de condutividade hidráulica quando comparado aos demais tratamentos.
\end{abstract}

Palavras-chave: manejo do solo; qualidade do solo; dinâmica da água no solo.

\section{ABSTRACT \\ SOIL PHYSICAL ATTRIBUTES OF A FERRALSOL UNDER DIFFERENT MANAGEMENT SYSTEMS}

The objective of this work was to evaluate the behaviour of soil physical attributes of a Ferralsol under different management systems. The experiment was conducted at the Centro Experimental Central, Instituto Agronômico (IAC), located at Campinas, State of São Paulo, Brazil, during the years of 19901991. The soil of the area is a Ferralsol, managed with no-tillage, chisel plow and conventional tillage. The experiment was conducted in the field in plots measuring $7.5 \times 15.0 \mathrm{~m}$, with three replications for each management treatment. Analyzed soil physical properties were: water retention, aeration capacity, available water capacity, bulk density and hydraulic conductivity as a function of the water content $(\theta)$, through the instantaneous profile method, with evaluation of the water redistribution the soil profile for the different management systems. It can be concluded that: the chisel plow treatment showed greater water retention in the surface layer because of its greater soil porosity; soil hydraulic conductivity did not show significant differences between the three soil tillage treatments studied at the surface layer, whereas for deeper layers no-tillage system presented larger values of hydraulic conductivity as compared to the other treatments.

Key words: soil management; soil quality; soil water dynamics.

( $\left.{ }^{1}\right)$ Parte da Tese de Doutorado em Agronomia (Solos e Nutrição de Plantas) do primeiro autor apresentada a ESALQ/USP. Recebido para publicação em 20 de janeiro de 2009 e aceito em 22 de dezembro de 2009.

$\left({ }^{2}\right)$ Instituto Agronômico (IAC), Centro de Pesquisa e Desenvolvimento de Solos e Recursos Ambientais, Caixa Postal 28, 13012-970 Campinas (SP). E-mail: omcastro@iac.sp.gov.br; sidney@iac.sp.gov.br (*) Autor correspondente.

$\left({ }^{3}\right)$ Doutorando em Engenharia para o Desenvolvimento Rural, Universidade de Santiago de Compostela, Escola Politécnica Superior, s/n, 27002, Espanha. Bolsista MAEC-AECID. E-mail. glecio.machado@rai.usc.es. 


\section{INTRODUÇÃO}

As propriedades físico-hídricas do solo são de fundamental importância para o desenvolvimento das culturas, pois estas propriedades coordenam o fluxo de gases e água no solo. Dessa maneira, o conhecimento de como essas propriedades interagem com os diferentes sistemas de preparo do solo pode auxiliar a prática de um manejo do solo sustentável, principalmente porque de acordo com KLEIN e LiBARDI (2002), pouco tem sido estudado sobre os processos de infiltração, redistribuição e suprimento de água às culturas em solos não saturados.

O preparo do solo na agricultura varia amplamente em termos de implementos utilizados, profundidade de trabalho e pela intensidade de movimentação, de acordo com o sistema de manejo adotado. Assim, os atributos físico-hídricos têm suas características naturais modificadas para estados que favorecem ou desfavorecem os cultivos, e sobretudo levando à formação de camadas diferenciadas ao longo do perfil do solo (Hillel, 1971; Roth et al., 1988; Eltz et al., 1989; Sarvasi, 1994; De Maria et al., 1999; Klein e Libardi, 2000; ToRmena et al., 2004). Por essas razões, as operações de preparo do solo alteram a dinâmica natural dos atributos físico-hídricos, não só pelo seu efeito sobre a estrutura (HILLEL, 1980; SARVASI, 1994; Klein e Libardi, 2000; Klein e Libardi, 2002; OtTONI FiLHO, 2003), mas também pela manutenção dos resíduos na superfície que podem minimizar as perdas de água por evaporação (PRIMAVESI et al., 2004).

O aumento de umidade do solo necessariamente não implica em mais água disponível às plantas, pois se a estrutura do solo resultante do manejo adotado aumentar a microporosidade, a água pode ficar mais fortemente retida, aumentando a força necessária para sua extração (LETEY, 1991). A nãomovimentação periódica do solo sob o sistema de plantio direto torna-o mais compactado que o sistema de plantio convencional, aumentando a retenção de água (Eltz et al., 1989; SidiRAS et al., 1983). Por outro lado, a cobertura morta no plantio direto reduz a evaporação (Vieira, 1981; Klein e Libardi, 2000; Primavesi et al., 2004), prolongando o período úmido do solo, fase em que a água está mais fracamente retida (Libardi, 1999; Klein e Libardi, 2002).

As mudanças na estrutura do solo em função do seu manejo propiciam também mudanças no fluxo de água, representando um problema para o manejo das culturas. Sharma e UeHARA (1968) descrevem que para os Latossolos, a condutividade hidráulica decresce rapidamente, em função do seu estado de agregação ou estruturação. $\mathrm{O}$ estudo da condutividade hidráulica do solo sobre os diferentes sistemas de manejo ajuda a compreensão do fluxo de água ao longo do perfil do solo. Deste modo, HiLLel (1971) e Roth et al. (1988) descrevem como a presença de camadas compactadas diminue a condutividade hidráulica do solo. Blevins et al. (1984) descrevem maior condutividade hidráulica do solo sob plantio direto com o aumento da profundidade, quando comparado a outros sistemas de manejo.

Dessa maneira, e tendo em vista as limitações ocasionadas pelos distintos sistemas de preparo do solo, este trabalho teve como objetivo avaliar o comportamento de propriedades físico-hídricas de um Latossolo Vermelho eutroférrico sob diferentes sistemas de manejo.

\section{MATERIAL E MÉTODOS}

Este estudo foi desenvolvido em Campinas (SP), Brasil, localizada na latitude $22^{\circ} 54^{\prime} 20^{\prime \prime}$ Sul, longitude $47^{\circ} 05^{\prime} 34^{\prime \prime}$ Oeste, com altitude média de 674 m. De acordo com a classificação de Köppen o clima da região é do tipo Cwa.

O solo da área é Latossolo Vermelho eutroférrico, textura muito argilosa (EMBRAPA, 2006), cultivado com milho (Zea mays L.) ou soja (Glycine max L.) no verão, em rotação à aveia-preta (Avena strigosa Schieb.) no inverno, desde o ano de 1983/1984, sob três sistemas de manejo: plantio direto (PD), arado escarificador (PE) e arado de discos (PC). As amostragens foram realizadas no ano agrícola de 1990/1991 após a colheita de milho (Zea mays L.) cultivar C-606 Cargill.

O experimento foi realizado em parcelas de 7,5 x 15,0 m, com três repetições para cada sistema de manejo. O preparo com arado de discos, aqui denominado preparo convencional (PC), foi realizado com arado reversível de três discos de $28^{\prime \prime}$ a uma profundidade de $20 \mathrm{~cm}$, seguido de duas passadas de grade niveladora. O preparo com escarificador (PE) consistiu no uso de arado escarificador de sete hastes tipo oblíqua, com espaçamento entre sulcos de $33 \mathrm{~cm}$ e profundidade de trabalho de $30 \mathrm{~cm}$. Após a escarificação fez-se a gradeação por duas vezes. O sistema plantio direto (PD) consistiu em semeadura do milho sem preparo prévio do solo, usando semeadeira de disco ondulado para corte da palha, disco duplo na linha da semente e facão sulcador para colocação do adubo a $10 \mathrm{~cm}$ de profundidade.

Após a colheita do milho foram abertas trincheiras de $1,5 \mathrm{~m}$ de profundidade e $1,0 \mathrm{~m}$ de largura em cada parcela, para determinação da densidade e retenção de água no solo nos diferentes sistemas de manejo, nas seguintes profundidades: 5 , $10,15,20,25,30,45,60,75,90$ e $120 \mathrm{~cm}$, utilizando 
anéis volumétricos de $100 \mathrm{~cm}^{3}$. As amostras foram saturadas por 48 horas em bandeja com água até dois terços da altura do anel. Em seguida foi determinada a massa da amostra saturada, e posteriormente, cada amostra foi submetida às tensões de 0,$0005 ; 0,002$; 0,005 e $0,006 \mathrm{MPa}$ em mesa de tensão e às tensões de $0,01,0,03,0,05,0,1,0,2,0,5,0,8$ e 1,5 MPa em câmara de Richards com placa porosa, pesando-se as amostras após equilíbrio em cada tensão. A densidade do solo e a umidade nos diferentes pontos da curva característica, foram determinadas de acordo com CAMARgo et al. (1986).

A capacidade de aeração (Car) e a capacidade de água disponível (Cad), foram determinadas de acordo com THOMASSON (1978), onde ambos os atributos são expressos em porcentagem de volume de solo amostrado (Equações 1 e 2):

$$
\begin{aligned}
& \text { Car }=\theta_{S}-\theta_{0,005 \mathrm{MPa}} \\
& \text { Cad }=\theta_{0,005 \mathrm{MPa}}-\theta_{1,5 \mathrm{MPa}}
\end{aligned}
$$

em que: $\theta_{S}$ é a umidade de saturação; $\theta_{0,005 \mathrm{MPa}}$ é o volume de água retido a $0,005 \mathrm{MPa}$ de sucção e $\theta_{1,5 \mathrm{MPa}}$ é o volume de água retido a $1,5 \mathrm{MPa}$ de sucção.

Posteriormente, em cada uma das parcelas de cada tratamento foram retirados os restos da cultura de milho (Zea mays L.), onde foi cravado um quadro de folha galvanizada de $3 \mathrm{~m} \times 3 \mathrm{~m}$ com $0,30 \mathrm{~m}$ de altura. Dentro desta parcela de $9 \mathrm{~m}^{2}$ foram instaladas três baterias de tensiômetros nas profundidades 10, 20, 30, 60, 90 e $120 \mathrm{~cm}$. A parcela foi inundada até que o tensiômetro a $120 \mathrm{~cm}$ de profundidade acusasse que o solo nesta camada estava saturado. A seguir, cortou-se o fornecimento de água, esperou-se acabar a água que ainda estava na superfície para, em seguida, cobrir toda a parcela com plástico e palha para se evitar as perdas de água por evaporação. Feito isto, deu-se início às leituras dos tensiômetros com intervalo de 1 hora até completar sete leituras, passando depois para intervalos de 12 horas e 24 horas até completar 17 dias, quando as leituras se estabilizaram. Esses dados permitiram determinar a condutividade hidráulica de acordo com metodologia descrita por Hillel et al. (1972), na qual a densidade do fluxo de água está relacionada com o gradiente de potencial total através da equação de Darcy:

$$
q=\frac{-K(\theta) \partial \psi}{\partial z}
$$

em que, substituída na equação da continuidade, fornece a equação diferencial geral que descreve o movimento da água no solo:

$$
\frac{\partial \psi}{\partial t}=\partial \psi / \partial z[K(\theta) \partial \psi / \partial z]
$$

em que: $K$ é a condutividade hidráulica do solo $(\mathrm{cm}$ dia $\left.{ }^{-1}\right)$, a qual é função da sua umidade $\theta\left(\mathrm{cm}^{3} \mathrm{~cm}^{-3}\right) ; \psi$ é o potencial total da água no solo $(\mathrm{cm}) ; z$ é a coordenada vertical de posição $(\mathrm{cm})$ e $t$ é o tempo (segundos). Integrando a Equação 3 em relação a $z$, a partir da superfície do solo até uma profundidade $L$, obtém-se:

$$
\int_{0}^{L}(\partial \theta / \partial t) d z[K(\theta) \partial \psi / \partial z]_{=L}-[K(\theta) \partial \psi / \partial z]_{=0}
$$

Como o solo se encontra coberto com plástico em $z=0$, o fluxo nesta profundidade é nulo e a equação se reduz a:

$$
\int_{0}^{L}(\partial \theta / \partial t) d z=[K(\theta) \partial \psi / \partial z]_{z=L}
$$

de onde se pode explicitar $K(\theta)$ :

$$
K(\theta)=\frac{\int_{0}^{L}(\partial \theta / \partial t) d z}{\partial \psi / \partial z}
$$

Assim, a condutividade hidráulica é obtida a partir da densidade de fluxo $q$, calculada pela integral da Equação 7 e do gradiente de potencial $\partial \psi / \partial z$.

Para o cálculo da condutividade hidráulica, as leituras do tensiômetro foram transformadas em potencial matricial $\left(\theta_{m}\right)$ pela Equação 8 .

$$
\psi_{h 2}=-12,6 H+h_{1}+h_{2}
$$

em que: $\psi_{h 2}$ é a tensão da água no solo na profundidade $h_{2}$, em $\mathrm{cm}$ de coluna d'água (cm.c.a); Hé a leitura da coluna de mercúrio $(\mathrm{cm})$; $h_{1}$ é a altura do reservatório de mercúrio ao solo $(\mathrm{cm}) ; h_{2}$ é a profundidade do tensiômetro no solo (cm).

Com os dados de retenção de água estabeleceu-se a equação de ajuste pelo modelo de Van Genuchten e Nielsen (1985) (Equação 9) para cada profundidade e, por intermédio desta equação, determinou-se o valor de umidade volumétrica $(\theta)$ para cada valor de potencial matricial $\left(\psi_{m}\right)$ gerado pelas leituras dos tensiômetros, ao longo do tempo $(t)$.

$$
\theta=\theta_{R}+\left\{\frac{\left(\theta_{S}-\theta_{R}\right)}{\left[1+(\alpha h)^{n}\right]^{7}}\right\}
$$

em que: $\theta$ é a umidade do solo na tensão $h ; \theta_{R}$ é a umidade residual; $\theta_{S}$ é a umidade de saturação; $\alpha$ é o valor de entrada de ar; $h$ é a tensão da água no solo; $m$ e $n$ são parâmetros empíricos. 
A partir dos valores de $\theta$ e $\psi_{m}$ para as diferentes profundidades $z(10,20,30,60,90$ e $120 \mathrm{~cm})$, nos diferentes tempos de drenagem $(t)$, chegou-se à determinação da condutividade hidráulica $K$ para cada profundidade e para diferentes valores de è por meio da Equação 7 e estabeleceu-se a relação de $\operatorname{LnK}$ versus $\theta$ por regressão linear, com a equação representativa da função $K(\theta)$ para cada profundidade conforme a Equação 10:

$$
K(\theta)=K_{0} \cdot e^{\gamma\left(\theta-\theta_{0}\right)}
$$

em que: $\gamma$ é o coeficiente angular da reta de $L n K$ versus $\theta, K_{0}$ a condutividade hidráulica do solo saturado e $\theta_{0}$ a umidade volumétrica de saturação.

A comparação dos dados obtidos foi realizada aplicando-se o teste de Duncan, usando o programa estatístico SANEST (Zonta e MAchado, 1984), comparando-se os três sistemas de preparo do solo: plantio direto $(\mathrm{PD})$, escarificação (PE) e preparo convencional (PC).

\section{RESULTADOS E DISCUSSÃO}

A figura 1 apresenta os valores da retenção de água no solo $\left(\theta, \mathrm{m}^{3} \mathrm{~m}^{-3}\right)$ para os distintos sistemas de manejo (PD, PE e PC) em profundidade $(5,10,15$, $20,25,30,45,60,75,90$ e $120 \mathrm{~cm}$ ). Percebe-se que até $25 \mathrm{~cm}$ de profundidade no PE ocorrem os maiores valores de retenção de água no solo nas tensões até $0,002 \mathrm{MPa}$, possivelmente porque este sistema de manejo tem maior macroporosidade, conforme descrito por Tormena et al. (2004). Os dados de densidade do solo $\left(\mathrm{kg} \mathrm{dm}^{-3}\right.$, Tabela 1) demonstram que nas camadas iniciais $(5,10$ e $15 \mathrm{~cm})$ no PE notamse os menores valores de densidade quando comparado aos demais sistemas de manejo, fato também constatado por outros autores (SARVASI, 1994; ToRMEnA et al., 2004). Todavia, em termos de retenção de água esse fato favorece maior retenção nessas camadas, principalmente em função da tensão aplicada (Figura 1). Esses resultados coincidem com os de SARVASI (1994), que também constatou maior retenção de água até $0,002 \mathrm{MPa}$ e menores valores de densidade do solo em Latossolo Vermelho manejado com arado escarificador, quando comparados a outros sistemas de manejo.

Nas tensões entre 0,005 e $1,5 \mathrm{MPa}$ correspondentes a capacidade de água disponível (THOMASSON, 1978), verifica-se que no PD verificamse os maiores valores de umidade com o aumento da tensão aplicada. Nesta faixa de tensão ( $\mathrm{MPa})$ predomina a ação dos meso e microporos que passam a regular a retenção da água, após a drenagem dos macroporos (Hillel, 1980; Klein e Libardi, 2000; Ottoni Filho, 2003). A $20 \mathrm{~cm}$ de profundidade, embora o PC seja mais compactado que o PD e o PE (Tabela 1), não resultou em diferenças com relação à retenção de água de água no solo. Havendo diferenças apenas nos pontos acima de $0,5 \mathrm{MPa}$ (Figura 1), faixa de retenção onde as plantas já podem entrar em murcha permanente (Hillel, 1980).

Nas demais profundidades, no PD ocorrem os maiores valores de retenção de água. Consequentemente, essa maior umidade contribui para maior condutividade hidráulica e térmica do solo (Klein e Libardi, 2000), propiciando maior desenvolvimento das culturas. Esses dados coincidem com os de Eltz et al. (1989) e de SidiRAs et al. (1983), que obtiveram em Latossolo Roxo até 5\% a mais de umidade no PD, significando maior disponibilidade de água às plantas. Essa maior retenção de água no PD se deve naturalmente à maior densidade do solo, com maior presença de microporosidade nas camadas superficiais e ao microclima desenvolvido pela manutenção da palha em superfície (Primavesi et al., 2004).

Pode-se destacar também que, pelos dados de densidade do solo (Tabela $1, \mathrm{~kg} \mathrm{dm}^{-3}$ ) apenas verificam-se diferenças significativas a 20 e $60 \mathrm{~cm}$ de profundidade. A $20 \mathrm{~cm}$ de profundidade ocorre diferenciação pelo teste de Duncan (5\%) no PC pela formação de uma camada compactada denominada pé-de-grade (CAMARGo, 1983). A $60 \mathrm{~cm}$ de profundidade o PD se diferenciou dos demais sistemas de manejo com maior valor de densidade do solo $(1,18$ $\left.\mathrm{kg} \mathrm{dm}^{-3}\right)$. Todavia, este valor de densidade descrito para o PD não comprometeu os resultados desse tratamento, uma vez que ocorrem maior retenção de água e capacidade de água disponível nesses sistemas nas camadas mais profundas.

Os dados referentes à capacidade de aeração (Car, Figura 2), demonstram que as maiores diferenças ocorrem a até $15 \mathrm{~cm}$ de profundidade, onde o PE tem os maiores valores de Car, o que confirma a predominância de macroporos, quando comparado aos demais sistemas de manejo (PD e PC). Nas demais profundidades, destaca-se o valor da Car para o PC a $20 \mathrm{~cm}(14,4 \%)$, situação em que ocorre a maior densidade do solo $\left(1,42 \mathrm{~kg} \mathrm{~m}^{-3}\right.$, Tabela 1) obtida entre os sistemas de manejo estudados. De maneira geral, os valores de Car observados para os diferentes sistemas de manejo estão acima do índice de 5\%, considerado crítico para a aeração por THOMASSON (1978), e também estão acima dos constatados por SARVASI (1994) trabalhando com Latossolo Roxo sob diferentes sistemas de manejo. 

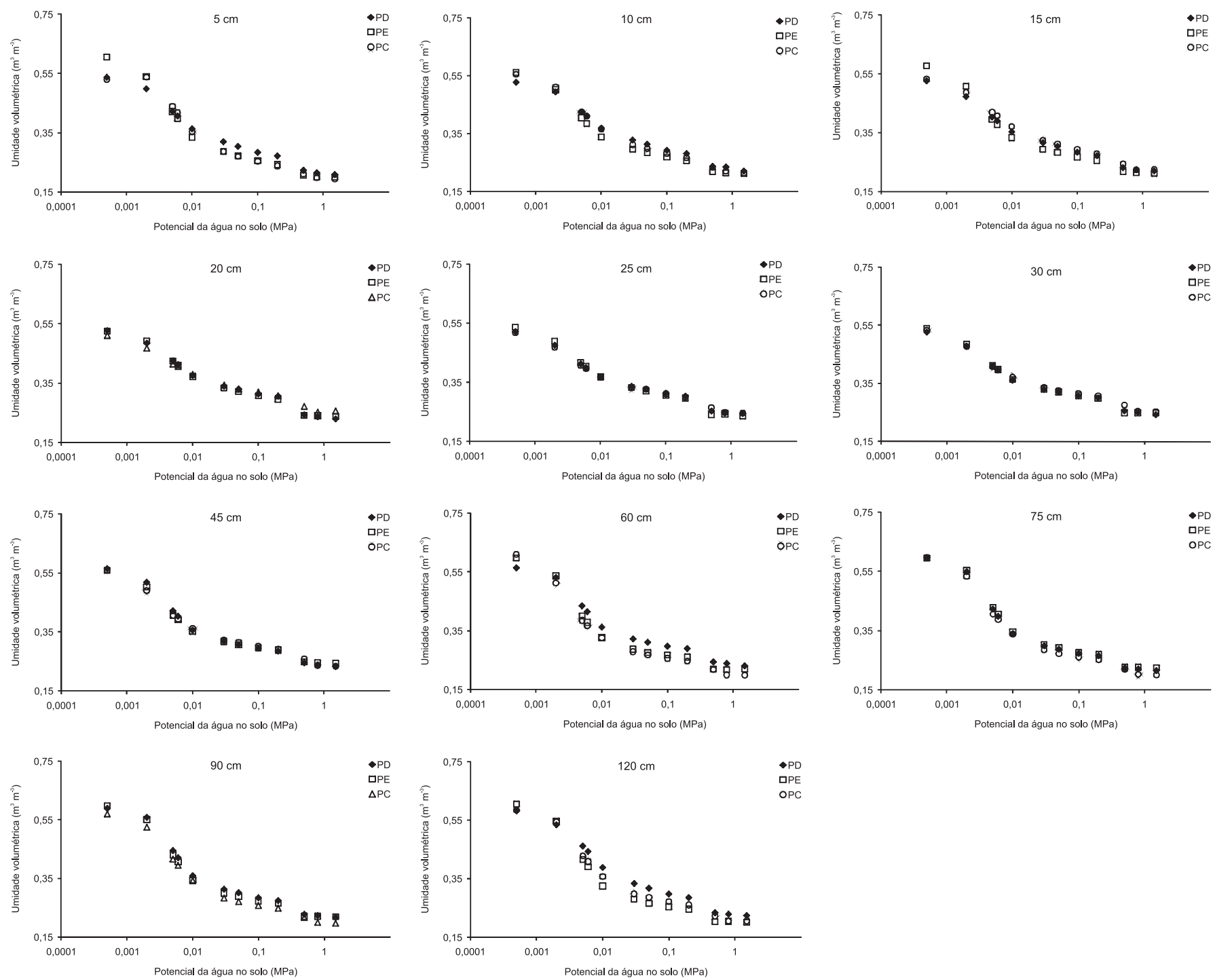

Figura 1. Retenção de água em função da profundidade $(5,10,15,20,25,30,45,60,75,90$ e 120 cm) e usos (PD, PE e PC) para o Latossolo Vermelho eutroférrico, em Campinas (SP). Cada símbolo representa o valor médio de 3 repetições.

Tabela 1. Comparação estatística dos dados de densidade do solo $\left(\mathrm{kg} \mathrm{dm}^{-3}\right)$ ao longo do perfil do solo e para os distintos manejos (PD, PE e PC)

\begin{tabular}{|c|c|c|c|c|c|c|c|c|c|c|c|}
\hline \multirow{2}{*}{ Sistemas de Preparo } & \multicolumn{11}{|c|}{ Profundidade $(\mathrm{cm})$} \\
\hline & 5 & 10 & 15 & 20 & 25 & 30 & 45 & 60 & 75 & 90 & 120 \\
\hline & \multicolumn{11}{|c|}{ Densidade do solo } \\
\hline & & & & & 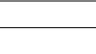 & $\mathrm{kg} \mathrm{dm}^{-}$ & & & & & \\
\hline PD & $1,26 a$ & $1,34 \mathrm{a}$ & $1,31 \mathrm{a}$ & $1,29 \mathrm{a}$ & $1,31 \mathrm{a}$ & $1,29 \mathrm{a}$ & $1,20 \mathrm{a}$ & $1,18 \mathrm{a}$ & $1,07 \mathrm{a}$ & $1,07 \mathrm{a}$ & $1,08 \mathrm{a}$ \\
\hline PE & $1,17 \mathrm{a}$ & $1,22 \mathrm{a}$ & $1,23 \mathrm{a}$ & $1,32 \mathrm{a}$ & $1,31 \mathrm{a}$ & $1,30 \mathrm{a}$ & $1,21 \mathrm{a}$ & $1,06 \mathrm{~b}$ & $1,07 \mathrm{a}$ & $1,04 \mathrm{a}$ & $0,96 \mathrm{a}$ \\
\hline PC & $1,18 \mathrm{a}$ & $1,29 a$ & $1,33 \mathrm{a}$ & $1,42 b$ & $1,32 \mathrm{a}$ & $1,33 a$ & $1,21 \mathrm{a}$ & $1,01 \mathrm{~b}$ & $1,01 \mathrm{a}$ & $0,99 a$ & $1,01 \mathrm{a}$ \\
\hline $\mathrm{CV} \%$ & 5,4 & 5,8 & 6,9 & 4,5 & 4,4 & 5,5 & 6,2 & 4,4 & 6,4 & 6,1 & 5,9 \\
\hline
\end{tabular}

Números seguidos pela mesma letra não diferem entre si, na mesma profundidade, a $5 \%$ de probabilidade (Duncan). 

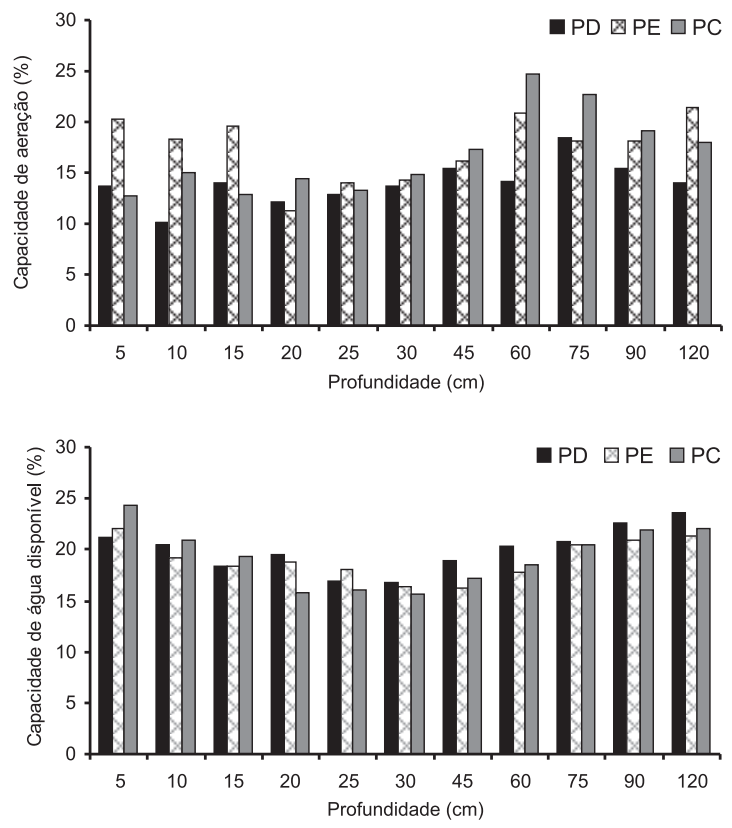

Figura 2. Capacidade de aeração (\%) e capacidade de água disponível (\%) para o Latossolo Vermelho eutroférrico nas diferentes profundidades $(5,10,15,20,25,30,45$, $60,75,90$ e $120 \mathrm{~cm}$ ) e para os distintos manejos do solo (PD, PE e PC). Cada barra representa o valor médio de três repetições.

Para a capacidade de água disponível (Cad, Figura 2), não se observam diferenças significativas entre os distintos sistemas de preparo. De maneira geral, pode-se destacar que o PD inicialmente tem menores valores de Cad, na camada superficial do solo, e com o aumento da profundidade, os maiores valores de Cad quando comparado aos demais sistemas de preparo. Todavia, deve-se ter em mente que os valores de Cad da maneira como foram calculados dão apenas uma idéia da dinâmica e disponibilidade de água às culturas (KLEIN e Libardi, 2000). Com a diminuição da macroporosidade e o aumento da microporosidade ocorre aumento da tensão com que a água é retida no solo (BAUMER e Bakermans, 1973; Klein e Libardi, 2000). Deve-se considerar ainda que, o valor do ponto de murcha permanente (1,5 MPa) utilizado para determinação da Cad pode ser atingindo antes deste ponto dependendo da cultura e da condição de perda de água.

$\mathrm{Na}$ figura 3 estão indicados os valores de umidade $\left(\theta, \mathrm{m}^{3} \mathrm{~m}^{-3}\right)$ em função do tempo de drenagem (horas) para os distintos sistemas de manejo (PD, PE e PC). Percebe-se que nas profundidades de 10 e 20 $\mathrm{cm}$ ocorre umidade de saturação no tempo zero, maior no PE confirmando mais uma vez sua eficácia para o aumento da macroporosidade do solo. No entanto, com o passar do tempo, PD tem os maiores valores de umidade, indicando maior estabilidade do sistema poroso (LIBARDI, 1999).
Nas profundidades de 20 e $30 \mathrm{~cm}$ ocorrem poucas diferenças entre os diferentes sistemas de manejo. Todavia, a partir de $60 \mathrm{~cm}$ de profundidade é evidente o maior armazenamento de água no PD quando comparado aos demais sistemas de manejo em estudo.

A relação entre a umidade $\left(\theta, \mathrm{m}^{3} \mathrm{~m}^{-3}\right)$ e o tempo (horas) ao longo do perfil (Figura 4), demonstra maior armazenamento de água após 1 hora cessada a saturação na seguinte ordem: PE, PD e PC. Entretanto, com o decorrer do tempo, no PD ocorrem os maior valores de umidade com determinada similaridade ao longo do perfil entre os distintos tempos. Percebe-se também que, a $60 \mathrm{~cm}$ de profundidade, no PE e o PC há os menores valores de umidade, enquanto PD mantém tendência de aumento da umidade a partir dessa profundidade de maneira homogênea, semelhante aos resultados constatados por SIDIRAs et al. (1984). A presença de camadas do solo com maior ou menor valor de umidade ao longo do perfil no PE e no PC sugere ocorrer uma descontinuidade estrutural que afeta o fluxo de drenagem ao longo do perfil (LiBARDI, 1999), ocasionada principalmente pela quebra da estrutura natural do solo durante as operações de preparo nesses sistemas.

Na figura 5, a condutividade hidráulica do solo determinada por meio do método de Hillel et al. (1972). Observa-se que até $30 \mathrm{~cm}$ de profundidade ocorre pouca variação nos valores de condutividade hidráulica $\left(\mathrm{cm} \mathrm{h}^{-1}\right)$ entre os distintos sistemas de manejo. Todavia, a partir de $60 \mathrm{~cm}$ de profundidade, é possível verificar que o PD se destaca sobre os demais sistemas, principalmente quando o movimento de água no solo é governado por pequenas tensões, concordando com os resultados de Blevins et al. (1984), que descrevem maior condutividade hidráulica do solo não saturado para o sistema de plantio direto, quando comparado com outros sistemas de manejo.

Sharma e Uehara (1968) descrevem ainda que, a condutividade hidráulica para os Latossolos, decresce rapidamente, quando pequenas tensões são aplicadas. Este fato, pode ser visualizado ao longo de todo o perfil do solo estudado e para os diferentes sistemas de preparo. De acordo com KLEIN e LibARDi (2002), essa redução na condutividade hidráulica se baseia no princípio de que na faixa de umidade de domínio dos macroporos a condutividade hidráulica é governada pelo potencial gravitacional e, na umidade governada pelos microporos, atua o potencial mátrico. Podese ressaltar ainda que, esse comportamento da condutividade hidráulica nos Latossolos próximos a saturação se deve principalmente em função de sua estrutura microagregada (RADULOVICH et al., 1992; Klein e LibaRdi, 2002). 

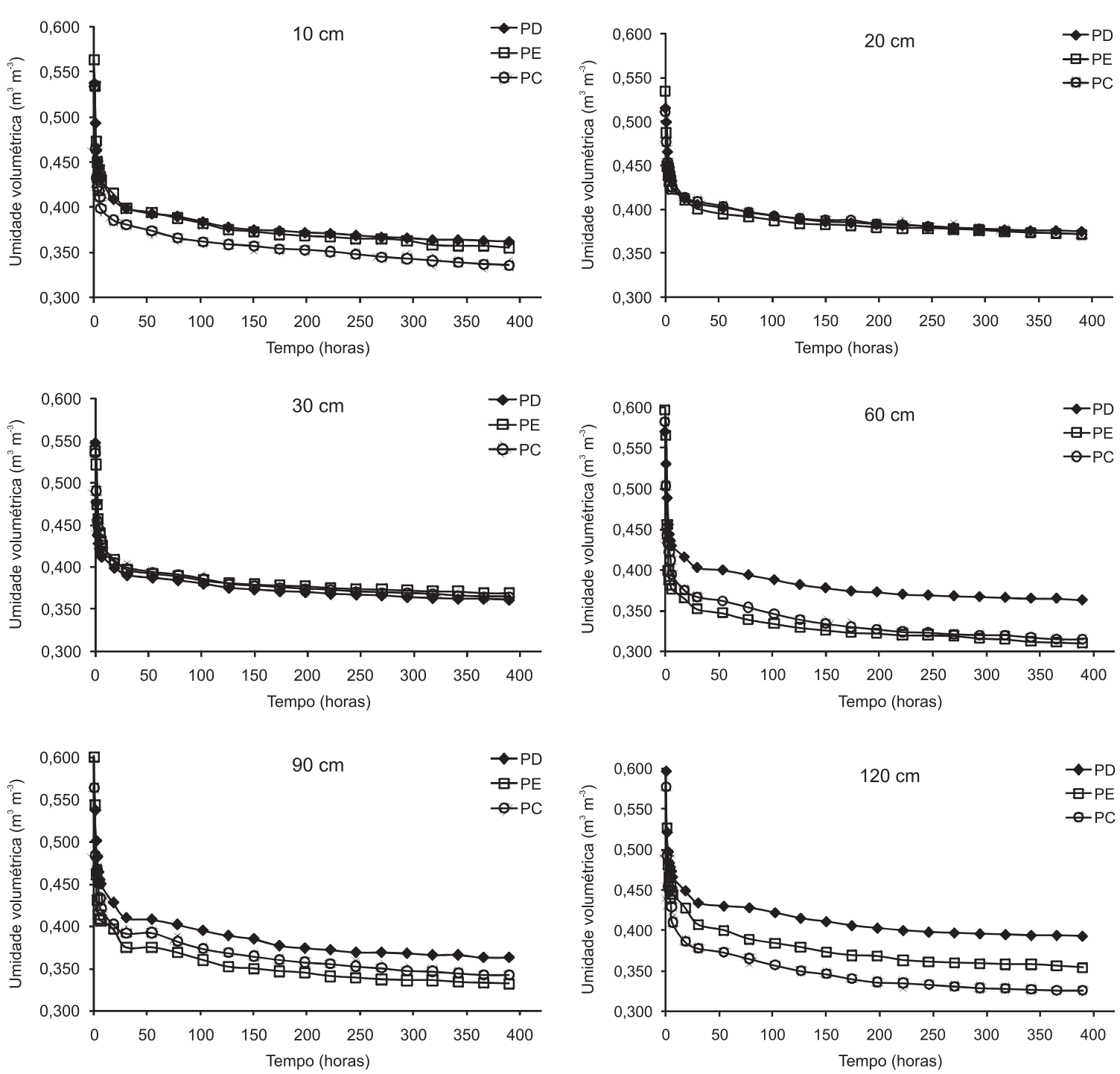

Figura 3. Umidade volumétrica $\left(\theta, \mathrm{m}^{3} \mathrm{~m}^{-3}\right)$, em função do tempo de drenagem para os três sistemas de preparo de solo (PD, PE e PC) e nas diferentes profundidades (10, 20, 30, 60, 90 e $120 \mathrm{~cm}$ ). Cada símbolo representa o valor médio de três repetições.

Vale a pena ressaltar a importância dos valores de densidade do solo (Tabela 1) sobre a condutividade hidráulica (Figura 5). Hillel (1971) e Rотн et al. (1988) descrevem que as camadas de solo com maiores valores de densidade $\left(\mathrm{kg} \mathrm{dm}^{-}\right.$ $\left.{ }^{3}\right)$ possuem respectivamente menores valores de condutividade hidráulica, principalmente influenciadas pelo manejo e cultivos sucessivos. Assim, no plantio direto ocorremos menores de valores de condutividade hidráulica na camada superficial, todavia, com aumento considerável desses valores com o aumento da profundidade, e principalmente, porque a densidade do solo é menor quando comparada aos demais sistemas de preparo.
A partir das relações entre a condutividade hidráulica $\left(\mathrm{K}, \mathrm{cm} \mathrm{h}^{-1}\right)$ e a umidade $\left(\theta, \mathrm{m}^{3} \mathrm{~m}^{-3}\right)$ apresentadas anteriormente, se estabeleceu a equação condutividade hidráulica saturada $\left(\mathrm{K}_{\theta}\right)$ para cada profundidade em cada tratamento de preparo, segundo o modelo exponencial apresentado na Equação 10 (Tabela 2).

Observa-se inicialmente, um valor $\mathrm{r}^{2}$ superior a 0,821 (Tabela 2), que garante boa acurácia da metodologia de estudo. Comparando-se os valores de condutividade hidráulica saturada $\left(\mathrm{K}_{\theta}\right)$ entre tratamentos, observa-se que no PD e PE houve menores valores que no $\mathrm{PC}$ na profundidade de $10 \mathrm{~cm}$; no PD a $20 \mathrm{~cm}$ observa-se o valor mais baixo, superando os demais a $30 \mathrm{~cm}$ de profundidade. 

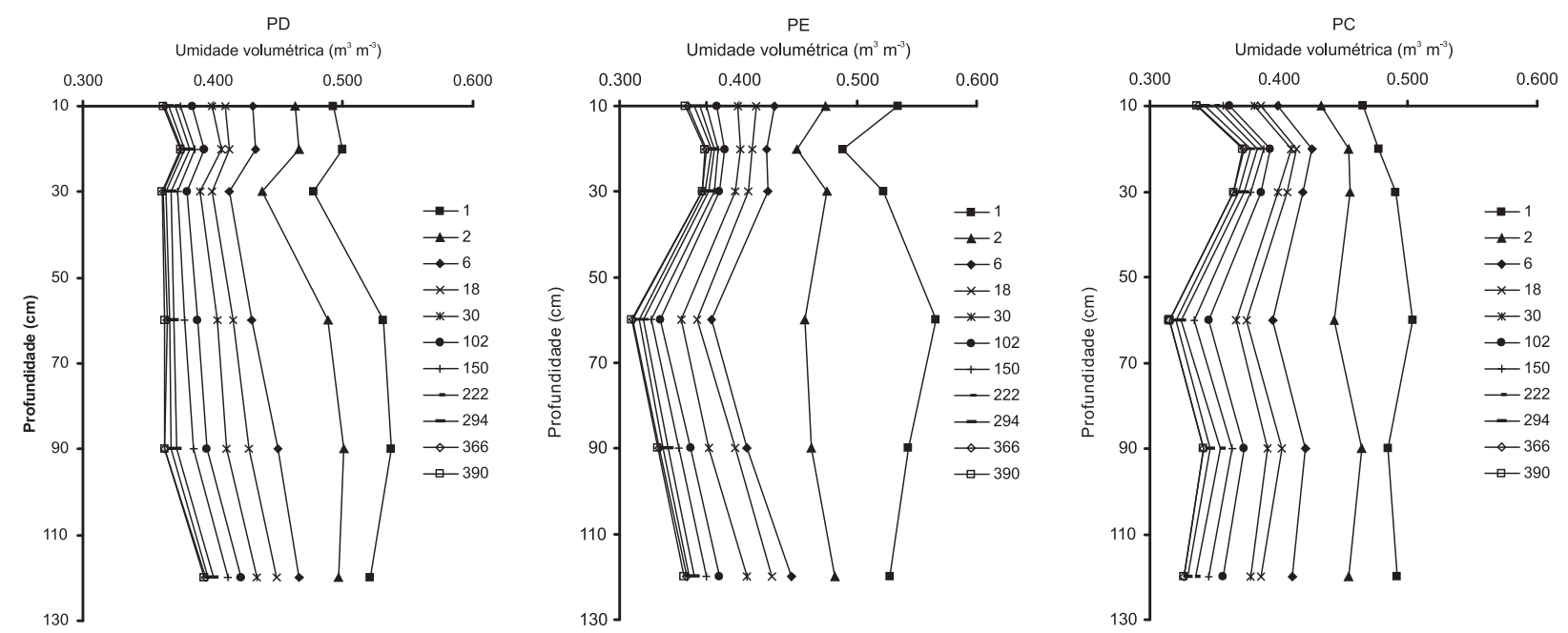

Figura 4. Perfil de umidade do solo (è, $\mathrm{m}^{3} \mathrm{~m}^{-3}$ ) para os diferentes sistemas de manejo do solo (PD, PE e PC) em diferentes tempos (horas). Cada símbolo representa o valor médio de três repetições.
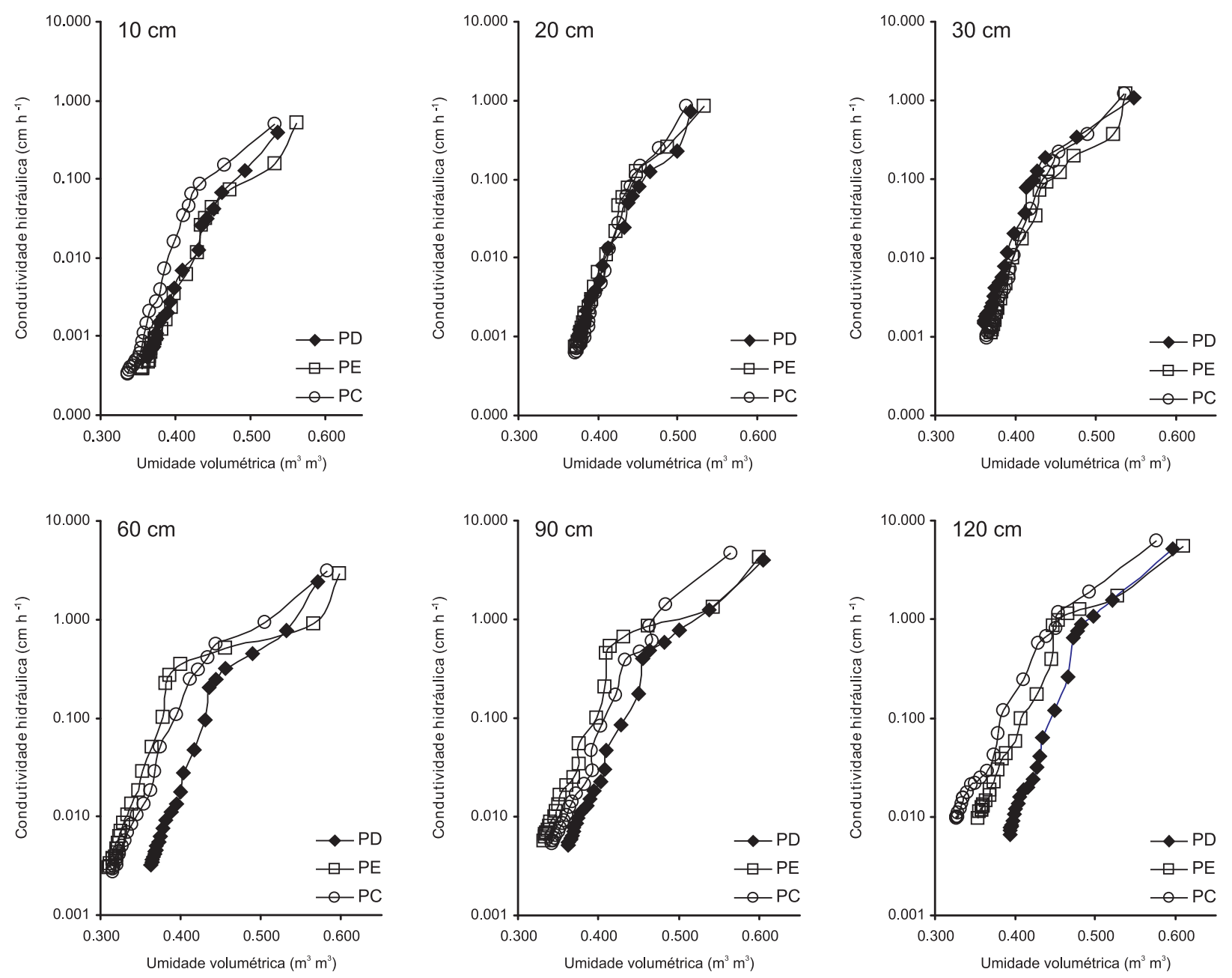

Figura 5. Condutividade hidráulica $\left(\mathrm{K}, \mathrm{cm} \mathrm{h}^{-1}\right)$ em função da umidade do solo $(\theta)$, nos diferentes sistemas de manejo (PD, PE e PC) e nas diferentes profundidades (10, 20, 30, 60, 90 e $120 \mathrm{~cm}$ ). Cada símbolo representa o valor médio de três repetições. 
Tabela 2. Parâmetros da Equação 10: $K_{\theta}$ e $r^{2}$ para cada uma das profundidades, e nos diferentes sistemas de preparo de solo

\begin{tabular}{lccccccc}
\hline \multirow{2}{*}{ Sistemas de Preparo } & & \multicolumn{5}{c}{ Profundidade } \\
\cline { 3 - 7 } & & 10 & 20 & 30 & 60 & 90 \\
\cline { 3 - 7 } PD & $\gamma$ & 46,776 & 52,537 & 52,005 & 39,091 & 35,712 & 46,051 \\
& $\theta$ & 0,537 & 0,551 & 0,551 & 0,581 & 0,606 & 0,604 \\
& $\kappa_{0}$ & 2,325 & 13,281 & 41,298 & 23,754 & 41,045 & 147,882 \\
& $\mathrm{r}^{2}$ & 0,983 & 0,976 & 0,927 & 0,936 & 0,960 & 0,961 \\
PE & $\gamma$ & 41,707 & 59,205 & 46,702 & 28,676 & 32,495 & 34,819 \\
& $\theta$ & 0,576 & 0,558 & 0,562 & 0,637 & 0,602 & 0,609 \\
& $\kappa_{0}$ & 4,891 & 58,085 & 14,584 & 71,223 & 61,656 & 97,608 \\
& $\mathrm{r}^{2}$ & 0,959 & 0,949 & 0,940 & 0,821 & 0,875 & 0,939 \\
PC & $\gamma$ & 45,183 & 59,545 & 47,978 & 30,749 & 35,821 & 30,297 \\
& $\theta$ & 0,588 & 0,542 & 0,561 & 0,613 & 0,615 & 0,630 \\
& $\kappa_{0}$ & 38,459 & 17,364 & 17,874 & 40,947 & 109,383 & 120,232 \\
& $\mathrm{r}^{2}$ & 0,917 & 0,967 & 0,936 & 0,917 & 0,959 \\
\hline
\end{tabular}

$\theta_{0}: \mathrm{cm}^{3} \mathrm{~cm}^{-3} ; \mathrm{K}_{0}: \mathrm{cm} \mathrm{h}^{-1}$

Esse menor valor de $\mathrm{K}_{\theta}$ para o PD nas camadas superficiais de certo modo contradiz a melhor infiltração descrita para esse sistema de manejo (Alves SOBRINHO et al., 2003) por meio da manutenção da palha em superfície que favorece ainda o controle da erosão hídrica (SILVA et al., 2000), ocorrendo principalmente em condições de solo saturado (RоTH et al.,1988; Arzeno,1990; Alves Sobrinho et al., 2003). Todavia, é preciso considerar que em um processo de infiltração a partir da superfície do solo, outros fatores interferem, principalmente o encrostamento superficial ocasionado pelo impacto das gotas de chuvas, como destacado por Roth et al. (1988) e ARZENo (1990), fato que não ocorre no PD, uma vez que este está protegido por meio dos restos das culturas anteriores mantidos em superfície (SILVA et al., 2000).
Dessa maneira, até que ocorra a saturação do solo, quem rege esse processo é a condutividade do solo não saturado (K) e por meio da tabela 3, verifica-se que até $60 \mathrm{~cm}$ de profundidade no PD houve superioridade acima de $50 \%$ em relação ao PC para a condutividade na umidade de capacidade de campo. Esse valor significa que, em PD, é necessário maior tempo para saturação do solo e, portanto, para se iniciar o escorrimento superficial, fato evidenciado em curvas de infiltração com chuva simulada por Roth e Meyer (1983), Derpsch et al. (1986) e Arzeno (1990); por esse motivo, em um segundo momento, notam-se melhores valores de condutividade hidráulica, quando comparado aos demais sistemas de preparo estudados.

Tabela 3. Valores de condutividade hidráulica na capacidade de campo (Kcc), em $\mathrm{cm} \mathrm{h}^{-1}$ para os três sistemas de preparo do solo, em cada profundidade

\begin{tabular}{|c|c|c|c|c|c|c|}
\hline \multirow{2}{*}{ Sistemas de Preparo } & \multicolumn{6}{|c|}{ Profundidade $(\mathrm{cm})$} \\
\hline & 10 & 20 & 30 & 60 & 90 & 120 \\
\hline & \multicolumn{6}{|c|}{ Kcc } \\
\hline & - & 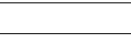 & 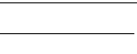 & & & \\
\hline PD & 0,00055 & 0,00102 & 0,00150 & 0,00326 & 0,00516 & 0,00668 \\
\hline PE & 0,00039 & 0,00072 & 0,00113 & 0,00306 & 0,00572 & 0,00976 \\
\hline PC & 0,00033 & 0,00062 & 0,00098 & 0,00279 & 0,00530 & 0,00953 \\
\hline
\end{tabular}


Pode-se dizer que as características físicohídricas do solo sob PD descrevem melhor desempenho em relação aos outros sistemas decorrente, em grande parte, pela manutenção da palha das culturas anteriores na superfície do solo. Em um primeiro momento, a cobertura morta reduz a taxa de evaporação, prolongando o tempo e o estado com que a água se mantém no solo (VIEIRA, 1981). Essa redução da evaporação se deve não só à ação da cobertura em si, que dificulta a saída do vapor d'água, mas também ao menor aquecimento do solo (KLein e Libardi, 2000) e redução da secagem superficial por ação do vento. Por outra parte, também é importante destacar que a palha em superfície diminui o escorrimento superficial, favorecendo que maior quantidade de água se infiltre no solo (Roth e Meyer,1983; Derpsch et al.,1986; ArZENo, 1990).

\section{CONCLUSÕES}

1. O plantio escarificado é o proporciona os maiores valores de retenção de água em superfície sob baixas tensões (até 0,002 $\mathrm{MPa}$ ), principalmente em função do aumento da porosidade neste sistema. Enquanto no sistema de plantio direto, verificam-se os maiores valores de retenção de água no solo em profundidade, quando comparado ao plantio escarificado e convencional.

2. A capacidade de aeração demonstra a maior macroporosidade presente no plantio escarificado em superfície $(20,27 \%)$. Em contrapartida, a capacidade de água disponível confirma maior disponibilidade de água no plantio direto em profundidade (23,65\%), ocasionadas principalmente pela manutenção da palha na superfície do solo.

3. Observando-se a condutividade hidráulica do solo não há diferenças para os três sistemas de preparo estudados na camada superficial. Já, em profundidade, o sistema de plantio direto proporciona os maiores valores de condutividade hidráulica quando comparado aos demais tratamentos.

\section{REFERÊNCIAS}

ALVES SOBRINHO, T.; VITORINO, A.C.T.; SOUZA, L.C.F.; GONÇALVES, M.C.; CARVALHO, D.F. Infiltração de água no solo nos sistema de plantio direto e convencional. Revista Brasileira de Engenharia Agrícola e Ambiental, v.7, p.191196, 2003.

ARZENO, J.L. Avaliação física de diferentes manejos de solo em latossolo roxo distrófico. 1990. 259f. Tese (Doutorado em Agronomia) ESALQ/USP, Piracicaba.
BAUMER, K.; BAKERMANS, W.A.P. Zero-tillage. Advances in Agronomy, New York, v.25, p.77-125, 1973.

BLEVINS, R.L.; SMITH, M.S.; THOMAS, G.W. Changes in soil properties under no-tillage. In: PHILLIPS, R.E.; PHILLIPS, S.H. (Ed.) No-tillage agriculture: principles and practices. New York: VNR, 1984. Cap.9, p.190-230.

CAMARGO, O.A. Compactação do solo e desenvolvimento de plantas. Campinas: Fundação Cargill, 1983. 44p.

CAMARGO, O.A.; MONIZ, A.C; JORGE, J.A.; VALADARES, J.M.A.S. Métodos de análise química, mineralógica e física de solos do Instituto Agronômico de Campinas. Campinas: Instituto Agronômico, 1986. 94p. (Boletim Técnico n. 106)

DE MARIA, I.C.; CASTRO, O.M.; SOUZA DIAS, H. Atributos físicos do solo e crescimento radicular de soja em Latossolo Roxo sob diferentes métodos de preparo do solo. Revista Brasileira de Ciência do Solo, v.23, p.703-709, 1999.

DERPSCH, R.; SIDIRAS, N.; ROTH, C.H. Results of studies made from 1977 to 1984 to control erosion by cover crops and tillage techniques in Paraná, Brazil. Soil \& Tillage Research, v.8, p. 253-263, 1986.

ELTZ, F.L.F.; PEIXOTO, R.T.G. \& JASTER, F. Efeitos de sistemas de preparo do solo nas propriedades físicas e químicas de um Latossolo Bruno Álico. Revista Brasileira de Ciência do Solo, v.13, p. 259-267, 1989.

EMBRAPA. Centro Nacional de Pesquisa de Solos. Sistema brasileiro de classificação de solos. 2.ed. Rio de Janeiro: Embrapa Solos, 2006. 306p.

HILLEL, D. Fundamentals of soil physics. New York, Academic Press, 1980. p.5-49; 50-75; 147-196.

HILLEL, D. The state of water in the soil. In: Soil and Water: Physical principles and processes. New York: Academic Press, 1971. p.49-77.

HILLEL, D.; KRENTOS, V.D.; STYLIANOU, Y. Procedure and test of an internal drainage method for measuring soil hydraulic characteristics in situ. Soil Science, v.114, p.395-400, 1972.

KLEIN, V.A.; LIBARDI, P.L. Condutividade hidráulica de um Latossolo Roxo, não saturado, sob diferentes sistemas de uso e manejo. Ciência Rural, v.32, p.645-653, 2002.

KLEIN, V.A.; LIBARDI, P.L. Faixa de umidade menos limitante ao crescimento vegetal e sua relação com a densidade do solo ao longo do perfil de um Latossolo Roxo. Ciência Rural, v.30, p.959-964, 2000.

LETEY, J. The study of soil structure: science on art. Australian Journal of Soil Research, v.29, p. 699-707, 1991.

LIBARDI, P.L. Dinâmica da água no solo, 2ed. Piracicaba: O autor, 1999. 497p.

OTTONI FILHO, T.B. Uma classificação físico-hídrica dos solos. Revista Brasileira de Ciência do Solo, v.27, p.211-222, 2003. 
PRIMAVESI, A.C.; PRIMAVESI, O.; CANTARELLA, H.; GODOY, R. Resposta da aveia branca à adubação em latossolo Vermelho-Amarelo em dois sistemas de plantio. Revista Brasileira de Zootecnia, v.33, p.79-86, 2004.

RADULOVICH, R.; SOLLINS, P.; BAVEYE, P. Bypass water flow through unsaturated microaggregated tropical soils. Soil Science of America Journal, v.56, p.721-726, 1992.

ROTH, C.H.; MEYER, B. Infiltrabilidade de um Latossolo Roxo distrófico durante o período vegetativo da soja sob o preparo convencional, escarificação e plantio direto. In: CONGRESSO BRASILEIRO DE CIÊNCIA DO SOLO, 19., Curitiba, 1983. Programa e Resumos... Campinas: SBCS, 1983. p.101-102.

ROTH, C.H.; MEYER, B.; FREDE, H.G. Effect of mulch rates and tillage systems on infiltrability and other soil physical properties of an oxisol in Paraná, Brasil. Soil \& Tillage Research, v.11, p.81-91, 1988.

SARVASI, F.O.C. Dinâmica da água, erosão hídrica e produtividade das culturas em função do preparo do solo. 1994. 149f. Dissertação (Mestrado em Agronomia) ESALQ-USP, Piracicaba.

SHARMA, M.L.; UEHARA, G. Influence of soil structure on water relation in low humic latosols: I. Water retention. Soil Science Society of America Journal, v.32, p.765-770, 1968.

SIDIRAS, N.; DERPSCH, R.; MONDARDO, A. Influência de diferentes sistemas de preparo do solo na variação da umidade e rendimento da soja, em Latossolo Roxo distrófico (Oxisol). Revista Brasileira de Ciência do Solo, v.7, p.103-106, 1983.

SIDIRAS, N.; ROTH, C.H.; FARIAS, G.S. Efeito da intensidade de chuva na desagregação por impacto de gotas em três sistemas de preparo do solo. Revista Brasileira de Ciência do Solo, v.8, p.251-254, 1984.

SILVA, V.R.; REINERT, D.J.; REICHERT, J.M. Resistência mecânica do solo à penetração influenciada pelo tráfico de uma colhedora em dois sistemas de manejo do solo. Ciência Rural, v.30, p.795-801, 2000.

THOMASSON, A.J. Towards and objective classification of soil structure. The Journal of Soil Science, v.29: p.38-46, 1978.

TORMENA, C.A.; FRIEDRICH, R.; PINTRO, J.C.; COSTA, A.C.S.; FIDALSKI, J. Propriedades físicas e taxa de estratificação de carbono orgânico num Latossolo Vermelho após dez anos sob dois sistemas de manejo. Revista Brasileira de Ciência do Solo, v.28, p.1023-1031, 2004.

VAN GENUCHTEN, M.T.; NIELSEN, D.R. On describing and predicting the hydraulic properties of unsaturated soils. Annales Geophysicae, v.3, p.615-628, 1985.

VIEIRA, M.J. Propriedades físicas do solo. In: Plantio direto no Estado do Paraná. Londrina: Fundação Instituto Agronômico do Paraná, 1981. p. 19-32. (Circular IAPAR, 23) 